\title{
Resultados asistenciales y económicos en los pacientes "periféricos" de medicina interna
}

\author{
Health care and economic outcomes \\ in "peripherical" patients of internal medicine
}

\author{
Eduardo Montero Ruiz, Laura Pérez Sánchez, José María Barbero Allende, \\ Rosa Agudo Alonso, Ángela Rebollar Merino, Joaquín López Álvarez \\ Servicio de Medicina Interna. Hospital Universitario Príncipe de Asturias, Alcalá de Henares (Madrid)
}

\begin{abstract}
Resumen
Fundamento y objetivo: El sistema tradicional de organización hospitalaria asigna un número de camas consecutivas, en una o varias unidades de hospitalización (plantas), a los diferentes servicios. Esta distribución permite una organización estable y circunscrita espacialmente, pero facilita la aparición de pacientes "periféricos".

Se analizó si los pacientes periféricos de Medicina Interna tienen peores resultados asistenciales y económicos que los ingresados en las camas propias del servicio.

Material y método: Estudio observacional retrospectivo que incluyó a los pacientes ingresados del 1 de enero al 15 de marzo y dados de alta antes del 1 de abril de 2015. Se compararon la estancia y mortalidad hospitalaria y los reingresos de los pacientes hospitalizados en las camas del servicio frente a los de los periféricos. Para el ajuste estadístico se utilizaron la edad, sexo, número de diagnósticos al alta y el índice de comorbilidad de Charlson.
\end{abstract}

Resultados: Participaron 1045 pacientes, el 27,8\% (IC 95\% 25,1 a 30,6) periféricos. La mortalidad en ambos grupos no difirió significativamente (OR 0,8 [IC 95\% 0,5 a 1,3]), pero sí los reingresos (OR 1,6 [IC 95\% 1 a $2,5] ; p=0,037$ ) y la estancia media (1 día [IC 95\% 0,1 a 1,8]; $p=0,034$ ). Esto supuso un incremento del $51,2 \%$ en los reingresos y del $12,2 \%$ en la estancia. El exceso en la estancia originó un aumento del gasto en $157.703,3 €$.

Conclusiones: Los pacientes periféricos de Medicina Interna tienen unos resultados asistenciales peores que los hospitalizados en las plantas propias del servicio, con un incremento asociado del gasto.

Palabras clave: Medicina Interna. Pacientes ingresados. Estancia hospitalaria. Reingreso hospitalario

\section{Introducción}

En el sistema tradicional de organización hospitalaria se asigna un número determinado de camas consecutivas, en una o varias unidades de hospitalización (plantas), a los diferentes servicios. Esta distribución permite una organización estable y circunscrita espacialmente, y facilita el trabajo en equipo, en especial entre el personal médico y el de enfermería. Su principal desventaja es que haya camas sin utilizar o, por el contrario, que su número sea insuficiente para hospitalizar a todos los enfermos asignados a ese servicio en un momento dado. Esta última posibilidad aboca a la aparición de pacientes "periféricos" o "ectópicos", que son aquellos a cargo de un servicio pero ingresados en camas asignadas a otro distinto. La aparición de estos pacientes periféricos distorsiona la dinámica del hospital y, en especial, el funcionamiento del servicio a cargo del enfermo

\begin{abstract}
Background and objective: Hospital management system assigns consecutive beds, in one or more inpatient units, to different medical or surgical services. This distribution allows a stable and spatially circumscribed organization, but facilitates the presence of "peripheral" patients.

It was studied whether peripheral Internal Medicine inpatients had worse health care and economic outcomes than those admitted to their own beds.

Materials and methods: Retrospective observational study involving patients admitted from January 1 to March 15 and discharged before 1 April 2015. Hospital readmissions, length of stay and in-hospital mortality of inpatients of Internal Medicine Service wards were compared against the peripheral ones. Used for statistical adjustement: age, sex, number of diagnoses at discharge and Charlson comorbidity index.

Results: The study included 1045 patients, $27.8 \%$ (95\% Cl 25.1 to 30.6) peripherals. Mortality in both groups did not differ significantly (OR 0.8 [95\% $\mathrm{Cl} 0.5$ to 1.3]), but they did readmissions (OR 1.6 [95\% $\mathrm{Cl} 1$ to 2.5]; $p=0.037)$ and length of stay ( $195 \% \mathrm{Cl} 0.1$ to 1.8$] ; p=0.034)$. This represented an increase of $51.2 \%$ in readmissions and $12.2 \%$ in length of stay. Excess of hospitalization days has led to an increase in spending $€ 157,703.3$.

Conclusions: Peripheral Internal Medicine patients have worse outcomes than inpatient care in their own beds, with an increase in associated costs. Key words: Internal Medicine. Inpatients. Length of stay. Hospital readmission.
\end{abstract}

y el del "invadido". Asimismo, puede conllevar riesgos para los propios pacientes.

En general, los gestores no suelen tomar medidas para evitar o paliar en lo posible la presencia de este tipo de pacientes y controlar sus efectos. Es probable que esto se deba a que, en la gestión de las camas hospitalarias, existe una tendencia a la desaparición de la asignación de camas a cada servicio y la formación de unidades de hospitalización polivalentes ${ }^{1}$, cuyo principal objetivo es el ahorro de costes. En los sistemas de salud actuales la productividad se ha incrementado a expensas de factores de seguridad ${ }^{2}$. Esto contrasta con otros campos de alto riesgo en los que la productividad es limitada por su adherencia a medidas de seguridad, las cuales tienen la máxima prioridad². Los servicios de Medicina Interna sufren con mucha frecuencia 
períodos de sobrecarga asistencial que originan un importante número de pacientes periféricos. Esta situación, habitual en nuestros hospitales, podría conllevar peores resultados asistenciales y riesgos para dichos enfermos, así como un incremento en los costes, al estar ubicados fuera de su "entorno natural", el servicio de Medicina Interna. Sin embargo, a pesar de su importancia para clínicos y gestores, no hemos encontrado trabajos que analicen estos problemas.

Nuestro objetivo fue estudiar si los pacientes periféricos de Medicina Interna tienen peores resultados asistenciales y económicos que los ingresados en las camas propias del servicio.

\section{Material y métodos}

El hospital donde se ha realizado el estudio está dotado con 450 camas para atender a una población casi exclusivamente urbana de 250.000 habitantes. Imparte docencia de pre y postgrado y está acreditado para la formación de residentes médicos y quirúrgicos. El servicio de Medicina Interna tiene asignadas 115 camas, repartidas entre tres secciones que ocupan tres unidades de hospitalización completas. En períodos de sobrecarga asistencial, los enfermos ingresan periféricos en diferentes plantas del hospital según la disponibilidad de camas, sin ningún otro criterio de selección adicional, siendo repartidos entre las tres secciones del servicio en función de los ingresos que ha tenido cada una de ellas cada día. Los facultativos de cada sección, junto con sus residentes, atienden a los periféricos además de a sus pacientes asignados habitualmente en la sección. No hay facultativos específicos para los periféricos, ni circuito diferente para las altas ni ninguna otra actividad.

El estudio, observacional retrospectivo, ha incluido a todos los pacientes con fecha de ingreso en Medicina Interna desde el 1 de enero hasta el 15 de marzo de 2015 y dados de alta antes del 1 de abril de 2015. Se ha realizado comparando la estancia y mortalidad hospitalarias y el número de reingresos de los pacientes hospitalizados en las camas propias de Medicina Interna con los de los hospitalizados periféricos. El reingreso fue definido como el nuevo ingreso por cualquier motivo y en cualquier servicio en el plazo máximo de 15 días desde el alta previa; no se diferenció entre ingreso urgente y programado porque este último tipo supone menos del $2 \%$ de los ingresos de pacientes de Medicina Interna. Para el ajuste estadístico se recogieron las siguientes variables: Edad, sexo, número de diagnósticos al alta y el índice de comorbilidad de Charlson (ICh), el cual está validado para su uso con bases de datos administrativas ${ }^{3}$, y con los pesos actualizados ${ }^{4}$. Además, en los pacientes periféricos se registró si el ingreso fue en camas de especialidades médicas o quirúrgicas, o en una planta abierta expresamente para este cometido (planta ex profeso), con personal de nueva contratación, por la presión asistencial, y que admitió ingresos desde el 13 de enero hasta el 18 de febrero. Con la excepción de esta última planta, el resto de las plantas del hospital no sufrieron ningún tipo de variación en su dotación de personal y medios. Por último, se registró la distribución por meses de los ingresos. Los datos fueron obtenidos del Conjunto Mínimo Básico de Datos (CMBD) del hospital, el cual admite hasta 13 diagnósticos codificados de acuerdo con la CIE-9-MC. Según los servicios administrativos del centro, el coste medio del día de estancia en Medicina Interna durante el año 2014 fue de 385,3 €. Para el cálculo de costes, se multiplicó este coste por el número de estancias.

\section{Análisis estadístico}

Las variables cuantitativas fueron descritas con la media y su intervalo de confianza del 95\% (IC 95\%), y se comprobó su distribución normal mediante la prueba de Kolmogorov-Smirnov; las cualitativas mediante el porcentaje y su IC 95\%. Por el tipo de trabajo y la naturaleza de los resultados se consideró suficiente una precisión de \pm 5 centésimas, por lo que fueron redondeados a un decimal. Las diferencias de medias se analizaron con la tde Student o la U de Mann-Whitney según fuera pertinente, las variables categóricas mediante la obtención de la Odds Ratio (OR). La estancia fue ajustada con un modelo de regresión lineal múltiple que incluyó todas las variables de ajuste estadístico. Las variables las introducimos en el modelo mediante el método de regresión por pasos. Los criterios de inclusión y exclusión de las variables en el modelo los fijamos en $p<0,05$ para la inclusión y $p>0,10$ para la exclusión. Los modelos finales los seleccionamos utilizando el criterio del cuadrado del coeficiente de correlación múltiple ajustado. El estudio multivariante de la mortalidad y de los reingresos fue llevado a cabo mediante regresión logística, introduciendo todas las variables de ajuste estadístico con el método de inclusión por pasos. Los criterios de inclusión y exclusión de las variables en los modelos los fijamos en $p<0,05$ para la inclusión y $p>0,10$ para la exclusión. La calibración de los modelos la valoramos por medio de la prueba de bondad de ajuste de Hosmer-Lemeshow. Para el análisis de tendencias el orden de los grupos ha sido Medicina Interna, plantas médicas, plantas quirúrgicas, planta ex profeso. Las tendencias en la estancia entre las diferentes plantas de hospitalización fueron estudiadas mediante el análisis de la varianza con contrastes polinómicos, y para la mortalidad y los reingresos con la prueba de tendencia lineal de MantelHaenszel. El nivel de significación estadística se estableció en $p<0,05$. Todos los cálculos fueron realizados con el paquete estadístico SPSS 15.0 (SPSS Inc. Chicago, Illinois, EEUU).

\section{Resultados}

El estudio incluyó un total de 1045 pacientes, de los que el 98,3\% (IC 95\% 97,5 - 99,1) fueron ingresos urgentes. El 72,2\% (IC 95\% 69,4 - 74,9) fueron hospitalizados en las camas de Medicina Interna y el 27,8\% (IC 95\% 25,1 - 30,6) periféricos: 4,3\% (IC 95\% 3,1 - 5,6) en plantas médicas, 15,5\% (IC 95\% 13,3 - 17,7) en quirúrgicas y 8\% (IC 95\% 6,4 - 9,7) en la planta abierta ex profeso (tablas 1 y 2). No se apreciaron diferencias en los ingresos periféricos entre el fin de semana y el resto de los días de la semana. La distribución por meses y unidades de hospitalización se muestra en la figura 1. Aunque aparecen algunas diferencias entre los diversos grupos en las variables de ajuste, no alcanzan significación estadística en ningún caso. Una vez realizado el ajuste con las variables de ajuste estadístico, la mortalidad de los enfermos ingresados en las camas de Medicina Interna y la de los periféricos no difiere significativamente (OR 0,8 [IC 95\% 0,5 - 1,3]; no significativo), pero sí lo hacen los reingresos (OR 1,6 [IC 95\% 1 - 2,5]; $p=0,037$ ) y la estancia media (diferencia 1 día [IC 95\% 0,1 - 1,8]; p=0,034). Esto su- 
Tabla 1. Resultados de los grupos Medicina Interna y Periféricos

\begin{tabular}{|c|c|c|c|c|}
\hline & Total & Medicina Interna & Periféricos & Dif/OR \\
\hline N & 1045 & 754 & 291 & $-0,1(-1,9-1,8)$ n.s. \\
\hline Edad; años (IC 95\%) & $79(78,2-79,8)$ & $79(78-79,9)$ & $78,9(77,3-80,5)$ & OR $1(0,8-1,3)$ n.s. \\
\hline Mujer; \% (IC 95\%) & $52,1(49-55,1)$ & $52(48,4-55,6)$ & $49,2(46,1-53,5)$ & $0(-0,3-0,4)$ n.s. \\
\hline ND (IC 95\%) & $11,1(10,9-11,2)$ & $11,1(10,9-11,3)$ & $11,1(10,8-11,4)$ & $-0,2(-0,5-0,2)$ n.s. \\
\hline ICh (IC 95\%) & $5,6(5,4-a) 5,8)$ & $5,7(5,4-5,9)$ & $5,5(5,2-5,8)$ & $0,9(-0,02-1,8)$ n.s. (p=0,055) \\
\hline Estancia; días (IC 95\%) & $9,9(9,5-10,3)$ & $9,7(9,2-10,1)$ & $10,6(9,7-11,4)$ & OR 0,8 (0,5 - 1,3) n.s. \\
\hline Exitus; \% (IC 95\%) & $12,4(10,4-14,4)$ & $13(10,6-15,4)$ & $11(7,4-14,6)$ & OR $1,6(1-2,4) p=0,041$ \\
\hline Reingresos \% (IC 95\%) & $9,4(7,6-11,1)$ & $8,2(6,3-10,2)$ & $12,4(8,6-16,2)$ & \\
\hline
\end{tabular}

ND: Número de diagnósticos al alta. ICh: Índice de comorbilidad de Charlson. IC 95\%: Intervalo de confianza del 95\%. Dif: Diferencia. OR: Odds Ratio. n.s.: No significativo.

pone un incremento del $51,2 \%$ en los reingresos y del $12,2 \%$ en la estancia. La media de pacientes periféricos ingresados cada día del estudio fue 35,6, lo que supone un $31 \%$ más sobre la capacidad del servicio.

Fig. 1. Distribución de pacientes por meses y plantas

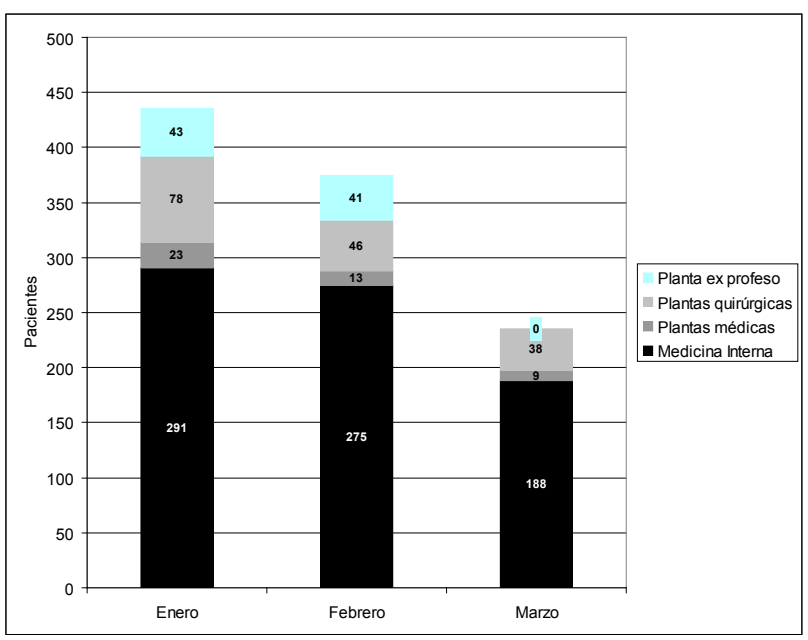

No se ha observado ninguna tendencia ni en la mortalidad ni en los reingresos, pero sí una tendencia lineal ascendente en la estancia ( $F=5,021 ; p=0,025)$.

Cada uno de los 291 pacientes periféricos tuvo un día de estancia más con respecto a los ingresados en Medicina Interna, originando 291 estancias extra. Asimismo, el grupo de periféricos ocasionó un 4,2\% más de reingresos que el de Medicina Interna, por lo que los 291 pacientes periféricos originaron 12,2 reingresos más que si hubieran estado ingresados en Medicina Interna.

Utilizando como referencia la estancia media de Medicina Interna (9,7 días), esos 12,2 reingresos implican otras 118,3 estancias. El exceso total de estancias acumulado por estos pacientes fue de 409,3. Aplicando el coste de día de estancia, los pacientes periféricos de Medicina Interna, en el periodo de estudio, implicaron un incremento del gasto en 157.703,3€.

\section{Discusión}

Nuestros hallazgos muestran que los pacientes periféricos de Medicina Interna tienen unos resultados asistenciales peores que los hospitalizados en las plantas propias del servicio, con un incremento asociado del gasto. Como el ingreso como periférico sólo depende de la disponibilidad de camas, el estudio podría considerarse muy próximo a uno aleatorizado. De hecho, ni la edad, el sexo ni la comorbilidad difieren significativamente entre los diferentes grupos, ésta última estudiada con el ICh y el número total de diagnósticos al alta que, conjuntamente, permiten valorar la complejidad y la comorbilidad médica ${ }^{5}$. No hubo traslados desde periféricos a las plantas de Medicina Interna. Debemos tener muy presente que la complejidad de un paciente se debe a una relación intrincada entre diversos factores, como son la enfermedad, el tratamiento, la familia o el comportamiento general de todos los estamentos e individuos que intervienen en su cuidado, más que a la coexistencia de varias enfermedades 6 . Por lo tanto, la complejidad podría estar influida por el hecho de ingresar en plantas con organizaciones diferentes o no habituadas a este tipo de pacientes y afectar a los resultados obtenidos.

Una forma indirecta de valorar la dificultad de manejo de los pacientes es mediante el análisis de la estancia, la cual además predice bien el coste asistencial ${ }^{7,8}$ y el riesgo de padecer sucesos adversos: $12 \%$ para $1-2$ días y $46 \%$ para más de 12 días 9 . Nuestros resultados muestran que los pacientes periféricos parecen presentar una mayor dificultad de manejo que quedaría reflejada en su mayor estancia. La tendencia observada entre los diferentes grupos nos indica que el problema parece radicar en las diferentes plantas y no en los médicos, ya que son los mismos para todas ellas. Según se van alejando las diversas plantas en sus características con respecto a la hospitalización de Medicina Interna, aumentan las dificultades, siendo las mayores en la planta de nueva creación, la cual precisa un rodaje para alcanzar la efectividad del resto de ellas. Este último hallazgo demuestra que las medidas precipitadas pueden ser contraproducentes.

El grado de organización y el desarrollo de las rutinas apropiadas de cualquier actividad, incluida la asistencial, es fundamental para obtener el fin deseado. Por ello, según nos alejamos de dichos parámetros los resultados empeoran. Así, se ha observado que los pacientes ingresados en Medicina Interna durante el fin de semana tienen mayor mortalidad ${ }^{10}$, se obtienen peores resultados en los pacientes operados los viernes ${ }^{11}$, existe una mayor dificultad de manejo en los pacientes ingresados en 
Tabla 2. Distribución y resultados de los pacientes periféricos

\begin{tabular}{|c|c|c|c|}
\hline & Pl. médicas & Pl. quirúrgicas & Planta ex profeso \\
\hline N & 45 & 162 & 84 \\
\hline Edad; años (IC 95\%) & $80,3(76-84,5)$ & $79(76,9-81,1)$ & $78,1(75,1-81,1)$ \\
\hline Mujer; \% (IC 95\%) & $48,9(40,2-58,8)$ & $48,8(41,1-56,5)$ & $50(39,3-60,7)$ \\
\hline ND (IC 95\%) & $11(10,2-11,7)$ & $11,3(10,9-11,6)$ & $10,9(10,3-11,5)$ \\
\hline ICh (IC 95\%) & $5,4(4,7-6,2)$ & $5,9(5,4-6,3)$ & $4,9(4,5-5,4)$ \\
\hline Estancia; días (IC 95\%) & $9,8(7,4-12,1)$ & $10,4(9,4-11,4)$ & $11,3(9,4-13,2)$ \\
\hline Exitus; \% IC 95\%) & $11,1(1,9-20,3)$ & $11,1(6,3-16)$ & $10,7(4,2-17,3)$ \\
\hline Reingresos \% (IC 95\%) & $11,1(1,9-20,3)$ & $13(7,8-18,1)$ & $11,9(5-18,8)$ \\
\hline
\end{tabular}

ND: Número de diagnósticos al alta. ICh: Índice de comorbilidad de Charlson. IC 95\%: Intervalo de confianza del 95\%.

servicios quirúrgicos que no son operados ${ }^{12}$, o que la mezcla de pacientes de diferentes especialidades quirúrgicas en las mismas habitaciones aumenta las infecciones de herida quirúrgica por gérmenes habituales de la otra especialidad y, además, las infecciones en general ${ }^{13}$.

Cabría esperar que a mayor dificultad mayor mortalidad, sin embargo no es así. Esto podría explicarse por una mayor implicación, a pesar de las dificultades, del personal médico y de enfermería en los pacientes más graves. También habría que tener en cuenta el efecto de la guardia médica en reducir la mortalidad, como ya se ha observado en el área quirúrgica ${ }^{14}$.

El reingreso hospitalario se considera un resultado adverso y un marcador de calidad asistencial ${ }^{15}$. Su precocidad, habitualmente antes de 30 días, está relacionada con la asistencia recibida durante el ingreso previo ${ }^{16}$. Por este motivo, hemos intentado asegurar más esta relación acortando a 15 días la definición de reingreso. Las razones por las que reingresan pacientes son múltiples y variadas, entre ellas su mayor complejidad ${ }^{17}$, la cual ya hemos comentado que también está relacionada con la organización y capacidades de las diferentes plantas.

Nuestros hallazgos muestran que el hecho de ingresar periférico acarrea un incremento importante de los costes económicos, a los que habría que añadir los posiblemente originados en todos aquellos servicios que se ven interferidos en su dinámica de trabajo por la presencia de los periféricos de Medicina Interna. Este hecho nos hace pensar que no está tan claro que la política de unidades de hospitalización polivalentes sea rentable económicamente.

Este trabajo tiene algunas limitaciones. Una de ellas es su diseño observacional retrospectivo. Asimismo, la obtención de datos a partir de bases administrativas podría hacer que éstos no fueran del todo fiables, si bien estas bases parecen tener una buena concordancia con los registros clínicos ${ }^{18}$. El hecho de estar realizado en un único hospital podría hacer que los resultados y conclusiones de este estudio pudieran no ser extrapolables a otros centros. Un inconveniente adicional es que no hemos encontrado bibliografía con la que poder comparar nuestros resultados.

Como conclusión podemos decir que la existencia de pacientes periféricos del servicio de Medicina Interna no solo empeora sus resultados asistenciales y económicos, sino que, además, po- dría afectar negativamente a otros servicios. Por ello, se deben tomar medidas, no improvisadas, que eviten 0 , al menos, palien esta situación. Los argumentos y resultados expuestos en este trabajo también pueden ser de utilidad a la hora de establecer unidades de hospitalización polivalentes.

\section{Bibliografía}

1. Grupo de Expertos. Unidad de enfermería de hospitalización polivalente de agudos. Estándares y recomendaciones. Agencia de Calidad del SNS. Ministerio de Sanidad y Política Social. 2009, (consultado el 10 de noviembre de 2015). Disponible en: http://www.msc.es/organizacion/sns/planCalidadSNS/docs/UHC.pdf

2. Amalberti R, Auroy $Y$, Berwick D, Barach P. Five system barriers to achieving ultrasafe health care. Ann Intern Med. 2005; 142: 756-64.

3. Deyo RA, Cherkin DC, Ciol MA. Adapting a clinical comorbidity index for use with ICD-9-CM administrative databases. J Clin Epidemiol. 1992; 45: 613-9.

4. Quan $\mathrm{H}, \mathrm{Li}$ B, Couris CM, Fushimi K, Graham P, Hider P, et al. Updating and validating the Charlson comorbidity index and score for risk adjustment in hospital discharge abstracts using data from 6 countries. Am J Epidemiol. 2011; 173: 676-82.

5. Martínez N, Gaminde I. Índices de comorbilidad y multimorbilidad en el paciente anciano. Med Clin (Barc). 2011; 136: 441-6.

6. Nardi R, Scanelli G, Corrao S, Iori I, Mathieu G, Cataldi Amatrian R. Co-morbidity does not reflect complexity in internal medicine patients. Eur J Intern Med. 2007; 18: 359-68.

7. Ferraris VA, Ferraris SP, Singh A. Operative outcome and hospital cost. J Thorac Cardiovasc Surg. 1998; 115:593-602.

8. Niskanen MM, Takala JA. Use of resources and postoperative outcome. Eur J Surg. 2001; 167: 643-9.

9. Kable AK, Gibberd RW, Spigelman AD. Adverse events in surgical patients in Australia. Int J Qual Health Care. 2002; 14: 269-76.

10. Marco J, Barba R, Plaza S, Losa JE, Canora J, Zapatero A. Analysis of the mortality of patients admitted to internal medicine wards over the weekend. Am J Med Qual. 2010; 25: 312-8.

11. Bottle A, Sanders RD. The "friday effect": Can epidemiology tell us when to operate? Can J Anaesth. 2015; 62: 852-6.

12. Montero Ruiz E, Barbero Allende JM, Melgar Molero V, Rebollar Merino Á, García Sánchez M, López Álvarez J. ¿Tienen los cirujanos más dificultades en la atención de los pacientes hospitalizados no intervenidos respecto a los intervenidos? Cir Esp. 2015; 93: 334-8.

13. Anwar M, Ferguson L, Awad Z, Ghufoor K. The impact of mixing surgical subspecialty patients on wound infection rates. Eur Arch Otorhinolaryngol. 2012; 269: 261-4.

14. Jones D, Egi M, Bellomo R, Goldsmith D. Effect of the medical emergency team on long-term mortality following major surgery. Crit Care. 2007; 11: R12.

15. Ashton CM, Wray NP. A conceptual framework for the study of early readmission as an indicator of quality of care. Soc Sci Med. 1996; 43; 1533-41.

16. Estrada D, López M, Martín E, Soriano RM, Grau JM. Reingresos hospitalarios no programados. Rev Calid Asist. 2014; 29: 59-69.

17. López Pérez J, López Álvarez J, Montero Ruiz E. Características diferenciales de los pacientes del Grupo Relacionado con el Diagnóstico (GRD) 541 que reingresan. Rev Calid Asist. 2015; 30: 237-42.

18. Guijarro R, Montes J, Sanromán C, Monreal M. for the RIETE Investigators. Venous thromboembolism in Spain. Comparison between an administrative database and the RIETE registry. Eur J Intern Med. 2008; 19: 443-6. 